\title{
Consumo de FDN y su efecto sobre la respuesta a la IATF en vacas del trópico de México
}

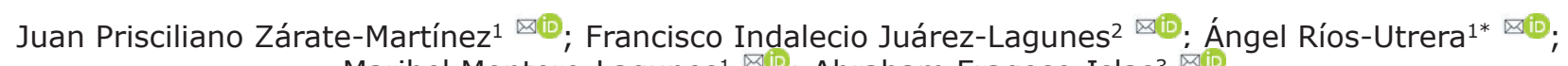
Maribel Montero-Lagunes ${ }^{1} \bowtie \mathbb{0} ;$ Abraham Fragoso-Islas $^{3} \otimes \mathbb{0}$.

\begin{abstract}
${ }^{1}$ Instituto Nacional de Investigaciones Forestales, Agrícolas y Pecuarias (INIFAP), Centro de Investigación Regional GolfoCentro, Campo Experimental La Posta. Paso del Toro, Medellín, Veracruz, México.

2Universidad Veracruzana, Facultad de Medicina Veterinaria y Zootecnia. Miguel Ángel de Quevedo S/N, Veracruz, México. 3Instituto Nacional de Investigaciones Forestales, Agrícolas y Pecuarias (INIFAP), Centro de Investigación Regional GolfoCentro, Sitio Experimental Las Margaritas. Hueytamalco, Puebla, México.

*Correspondencia: rios.angel@inifap.gob.mx
\end{abstract}

Recibido: Agosto 2020; Aceptado: Agosto 2021; Publicado: Noviembre 2021.

\section{RESUMEN}

Objetivo. Evaluar el comportamiento reproductivo de vacas cruzadas del trópico de México alimentadas con dos niveles de fibra detergente neutro (FDN), induciéndolas a ovular con un protocolo hormonal e IATF. Materiales y métodos. Se utilizaron 27 vacas multíparas. Veintiún días antes del parto, las vacas se asignaron a dos tratamientos: $T 1=4.5 \mathrm{~kg}$ de concentrado/vaca/d y T2 $=3.0 \mathrm{~kg}$ de concentrado/ vaca/d, y forraje ad libitum en ambos tratamientos. Resultados. Las vacas del T2 consumieron más FDN ( $p<0.05$ ) que las vacas del T1 (38 vs 44\% de la dieta). El incremento en el consumo de forraje, hasta donde la FDN permitió, logró compensar el aporte de EM y PM, siendo similares para ambos tratamientos durante los primeros 15 días antes del parto. Las vacas del T2 intentaron compensar el déficit de nutrientes consumiendo más forraje (6.25 vs $7.37 \mathrm{~kg}$ de MS/d). La PM siempre fue deficiente durante la lactación para T1 y T2. En las vacas del T2, la deficiente PM repercutió en menor contenido de $\mathrm{N}$ en leche. Las vacas del T1 perdieron menos condición corporal; esto se reflejó en una mayor $(p<0.05)$ tasa de gestación a primer servicio $(75.2$ vs $42.8 \%)$, aunque no produjeron más leche que las del T2. Conclusiones. La disminución de la FDN en la dieta de 44 a 38\% y la aplicación del protocolo hormonal de IATF disminuye el período abierto a 105 d en vacas lactando en el trópico.

Palabras clave: Consumo voluntario; fertilidad; periodo de transición; protocolo hormonal; sistema doble propósito; vacas cruzadas (Fuente: $C A B$ ).

\section{ABSTRACT}

Objective. To evaluate the reproductive performance of crossbred cows in the tropics of Mexico fed two levels of neutral detergent fiber (NDF), eliciting them to ovulate with a hormonal protocol and fixed-time artificial insemination (FTAI). Material and methods. Twenty-seven multiparous cows were used. Twenty-one days before calving, cows were assigned to two treatments: $T 1=4.5 \mathrm{~kg}$ of concentrate $/ \mathrm{cow} / \mathrm{d}$ and $\mathrm{T} 2=3.0 \mathrm{~kg}$ of concentrate/cow $/ \mathrm{d}$, and forage ad libitum in both treatments.

Results. Cows assigned to T2 consumed more NDF $(p<0.05)$ than cows assigned to T1 (38 vs $44 \%$

Como citar (Vancouver).

Zárate-Martínez JP, Juárez-Lagunes FI, Ríos-Utrera Á, Montero-Lagunes M, Fragoso-Islas A. Consumo de FDN y su efecto sobre la respuesta a la IATF en vacas del trópico de México. Rev MVZ Córdoba. 2022; 27(1):e2121. https://doi.org/10.21897/rmvz.2121 
of the diet). The increment in forage consumption, up to the NDF allowed, compensated the provision of metabolizable energy (ME) and protein (MP), which were similar in cows of both treatments during the 15 days before calving. Cows fed with T2 tried to compensate the nutrients deficit consuming more forage ( $6.25 \mathrm{vs} 7.37 \mathrm{~kg}$ of DM/d). The MP was always deficient during lactation for T1 and T2 cows. In T2 cows, the MP deficiency resulted in less milk nitrogen content. Cows assigned to T1 lost less body condition, which resulted in higher $(p<0.05)$ pregnancy rate at first service $(75.2 \mathrm{vs}$ $42.8 \%$ ), although they did not produce more milk than T2 cows. Conclusions. NDF reduction in the ration from 44 to $38 \%$ and the application of the FTAI hormonal protocol reduce the open period to $105 \mathrm{~d}$ in lactating cows in the tropics.

Keywords: Dual-purpose system; feed intake; fertility; crossbred cows; hormonal protocol; transition period (Source: $C A B$ ).

\section{INTRODUCCIÓN}

La ganadería de doble propósito con vacas cruzadas en el trópico húmedo de México basa su alimentación en dietas casi exclusivas de forraje, tanto en la etapa preparto como posparto. En ciertos casos, estas dietas son complementadas con alimentos concentrados después del parto. Se sabe que existe una etapa prioritaria de atención de la vaca tres semanas antes y tres semanas después del parto, denominando a este período "transición". Sin embargo, en la mayoría de los sistemas doble propósito esta etapa es totalmente ignorada.

Este periodo se caracteriza por una disminución del consumo de alimento, el cual se recobra de una a dos semanas después del parto. Esta caída del consumo de alimento genera un desbalance nutricional entre los requerimientos nutricionales y los nutrientes consumidos, ocasionando principalmente un desbalance energético negativo en el inicio de la lactación.

Para disminuir los factores de riesgo del período de transición, es importante lograr una progresiva adaptación del rumen (en especial con aquellas dietas donde existen desafíos de cantidad y calidad de alimento) e implementar estrategias de suplementación vitamínico-mineral o con nutrientes antioxidantes que mitiguen el estado de inmunosupresión y el estrés metabólico y oxidativo en la gestación y lactación y, de esta manera, prevenir estos problemas, y así lograr un impacto favorable sobre el comportamiento reproductivo y productivo (1).

La producción de leche bovina en el trópico se basa en el pastoreo $y$, por lo tanto, la calidad del forraje tiene un efecto directo sobre la producción y reproducción, ya que es la principal fuente de energía y proteína. La cantidad de materia seca (MS) de forraje consumida es el factor más importante que regula la producción de bovinos en pastoreo (2). La composición química de un forraje determina la calidad nutricional $y$, por ende, su consumo y valor en la producción animal (3), por lo que el consumo voluntario se debe conocer o predecir para determinar la proporción de los requerimientos que pueden ser cubiertos vía forrajes tropicales y así se pueda calcular la cantidad de concentrado complementario necesario por día.

Es necesario tener un claro entendimiento de los factores que regulan a corto plazo (cantidad consumida) y a largo plazo (grasa corporal), el apetito y el consumo voluntario (CV) del alimento, el cual está controlado por el estado fisiológico en el que el animal se encuentra. Con este trabajo se pretende evaluar el comportamiento reproductivo de vacas cruzadas del trópico de México alimentadas con dos niveles de fibra detergente neutro (FDN), induciéndolas a ovular con un protocolo hormonal e IATF.

\section{MATERIALES Y MÉTODOS}

Localización. El estudio se realizó de agosto de 2017 a febrero de 2019 en el Campo Experimental La Posta del INIFAP, ubicado en Paso del Toro, Veracruz, México, en el kilómetro 22.5 de la carretera federal Veracruz-Córdoba. La posición geoespacial comprende los paralelos $19^{\circ} 02^{\prime}$ de Latitud Norte y $96^{\circ} 08^{\prime}$ de Longitud Oeste. La altura sobre el nivel del mar es de $16 \mathrm{~m}$.

Ambiente. El clima de la región es Aw calientesubhúmedo con temperatura, precipitación pluvial y humedad relativa promedio anual de $25^{\circ} \mathrm{C}, 1461 \mathrm{~mm}$ y $75 \%$, respectivamente (4).

Animales. De un hato lechero de 100 vacas Holstein $x$ Cebú en sistema de doble propósito en 
pastoreo en clima tropical, se utilizaron las vacas multíparas gestantes programadas a parir en la época de lluvias (de junio a diciembre) del 2017. Veintisiete vacas ingresaron al experimento 30 días antes del parto y se alojaron en corrales individuales hasta los 70 días posparto.

Manejo de animales pre-tratamientos. Del día 30 al día 15 del período preparto, a todos los animales se les ofreció una cantidad fija de concentrado $(2 \mathrm{~kg}$ ) con $16 \%$ de PC y $70 \%$ de TND y heno de pasto Pangola (Digitaria decumbens) a libertad.

Tratamientos. Quince días antes de la fecha probable de parto, las vacas se asignaron aleatoriamente a dos tratamientos: $\mathrm{T} 1=4.5$ $\mathrm{kg}$ de concentrado/vaca/d y T2=3.0 kg de concentrado/vaca/d, en forma alternada, hasta completar 14 y 13 repeticiones en T1 y T2, respectivamente. En la Tabla 1 se presenta la formulación del concentrado. El forraje se ofreció a libertad y su consumo se determinó por diferencia entre lo ofrecido y el sobrante en 24 horas, de acuerdo con el requerimiento nutricional de las vacas, estimado con el Cornell Net Carbohydrate and Protein System (CNCPS) (5) con base en la producción de leche.

Tabla 1. Formulación del concentrado para los dos tratamientos experimentales de vacas de doble propósito en el trópico.

\begin{tabular}{cc}
\hline Ingrediente & Porcentaje \\
\hline Maíz molido & 43.5 \\
Sorgo molido & 21.0 \\
Salvado de trigo & 12.0 \\
Pasta de soya & 10.0 \\
Melaza & 10.0 \\
Vitaminas y minerales Beef Mass & \\
Aceite de soya & 2.0 \\
Urea grado alimenticio & 1.0 \\
\hline
\end{tabular}

Manejo de animales. Al parir, las vacas se ordeñaron una vez al día, en la mañana, con el apoyo del becerro. Ocho horas después del ordeño, se permitió que los becerros mamaran a libertad durante una hora. El peso corporal de las vacas y sus becerros, así como la condición corporal (CC; escala 1 a 9) de las vacas, se midió cada 14 días. La producción de leche se calculó diariamente, mientras que la composición de la leche (grasa, proteína y lactosa) se estimó cada 28 días con un analizador de leche ultrasónico marca Milkotronic Ltd, modelo LACTOSCAN S. 8900 Nova, Zagora, Bulgaria.
Análisis de composición química de los alimentos. El contenido de nutrientes en la dieta se determinó tomando cada 3 meses muestras de forraje y concentrado ofrecidos durante el período experimental, para realizar análisis de composición química. Para concentrado y forraje, se tomaron dos muestras de $500 \mathrm{~g}$ c/u, una muestra se secó a $100^{\circ} \mathrm{C}$ durante 24 h para determinar contenido de $\mathrm{MS}$; la otra se secó a $55^{\circ} \mathrm{C}$ hasta obtener peso constante. Esta última se molió en molino Wiley (Model 4, Arthur H. Thomas Co., Philadelphia, PA, USA) con malla de $1 \mathrm{~mm}$. Se determinó MS, cenizas, grasa cruda, proteína cruda y las fracciones de FDN, FDA y lignina (6), así como las fracciones de nitrógeno mediante el método estandarizado (7), de acuerdo con la estructura del CNCPS (8).

Estimación de nutrientes y requerimientos nutricionales. Para estimar el aporte de nutrientes y los requerimientos nutricionales de las vacas en los dos tratamientos, se usó el programa CNCPS V 6.5.5.1. Para el seguimiento diario del comportamiento nutricional de las vacas por tratamiento, se consideraron variables ambientales (temperatura ambiental y humedad relativa), de manejo (estabulación en corrales individuales), de grupo racial (cruzadas Holstein x Cebú), fisiológicas (peso y condición corporal, edad al parto, número de partos, producción y composición de leche) y del alimento (consumo de concentrado y forraje, y composición química nutricional del concentrado y forraje). La información se agrupó en periodos de 15 días: 15 días antes del parto y 15, 30, 45 y 60 días posparto.

Las variables estudiadas en estos períodos fueron: cambios de peso y condición corporal, consumo de concentrado, forraje y fibra detergente neutro (FDN), requerimientos de energía y proteína de mantenimiento, reservas, gestación (antes del parto) y lactación (después del parto) para los efectos de tratamiento. Las comparaciones entre T1 y T2 en cada período se realizaron con la prueba $t$ de student. Las implicaciones nutricionales en estos períodos se relacionaron con el inicio de la actividad ovárica posparto y el comportamiento reproductivo subsecuente en las vacas de cada uno de los tratamientos.

Reinicio de la actividad ovárica y manejo reproductivo. Para evaluar la dinámica folicular de las vacas durante los tratamientos, se tomaron imágenes (ultrasonido bug, marca BCF Innovative Imaging, con escáner de $7.5 \mathrm{MgHz}$ ) cada tercer día a partir del día 30 posparto hasta 
el día en que se presentó un folículo con un diámetro $\geq 10 \mathrm{~mm}$. A las vacas del T1, a partir de la aparición de dicho folículo, o a más tardar a partir del día 70 posparto, el cual se consideró como el día 0 , se les indujo el estro y la ovulación con un protocolo que constó de un CIDR con $1.9 \mathrm{~g}$ de progesterona $+2 \mathrm{mg}$ de benzoato de estradiol. El CIDR se retiró a los ocho días y se aplicó una inyección IM con 25 mg de PGF $_{2 a}, 0.5$ $\mathrm{mg}$ de cipionato de estradiol y 400 UI de eCG. Las vacas se pintaron y en el día 10 ( $58 \mathrm{~h}$ posteriores al retiro del CIDR) se realizó la inseminación artificial de vacas despintadas y pintadas (60 h posteriores al retiro del CIDR) y se aplicaron 2 $\mu \mathrm{g}$ de GnRH. Los becerros se destetaron por 72 h (destete temporal), comenzado en el momento de remoción del CIDR (día 8) hasta el día de la IATF (día 10). El manejo reproductivo de las vacas del T2 fue similar al de las vacas del T1.

Variables reproductivas. Para las variables reproductivas se aplicó un diseño completamente al azar. Las variables estudiadas fueron: días abiertos, intervalo entre partos, número de servicios por concepción y tasa de gestación a primer servicio. La tasa de gestación a primer servicio se consideró como una variable binaria; por lo tanto, cuando una vaca resultó gestante al primer servicio de IA dicha variable se registró como 1 , en caso contrario (vaca no gestante), se registró como 0 .

Análisis estadístico de variables reproductivas. Número de servicios por concepción y tasa de gestación a primer servicio se analizaron con PROC GENMOD del programa SAS (9). Para servicios por concepción, se especificó una distribución Poisson en la declaración del modelo, mientras que en el análisis estadístico de la tasa de gestación a primer servicio se especificó una distribución binomial y se usó una función liga logit. Días abiertos e intervalo entre partos se analizaron con el procedimiento GLM de SAS (9). En todos los casos, el modelo estadístico incluyó los efectos fijos de tratamiento, año de parto, época de parto y edad de la vaca al parto, excepto para días abiertos e intervalo entre partos, ya que no resultó significativa $(p>0.05)$ en análisis preliminares.

\section{RESULTADOS}

En la Tabla 2 se presentan las condiciones nutricionales de las vacas, por tratamiento, en el período correspondiente a 15 días antes del parto. Las vacas tuvieron condiciones fisiológicas muy similares al inicio del estudio debido a que, además de uniformizar por fecha probable de parto, se consideró la edad, el número de lactancia, peso y condición corporal de las vacas para que fueran lo más comparables por tratamiento. La similitud en las condiciones fisiológicas permitió observar consumos de MS muy similares entre tratamientos, a excepción del consumo de concentrado que estuvo experimentalmente controlado, ya que a las vacas del T1 se les ofreció más que a las vacas del T2 (4.5 vs $3.0 \mathrm{~kg} / \mathrm{vaca} / \mathrm{d})$. La repercusión en menor consumo de concentrado de las vacas del T2 es que intentaron compensar el déficit de nutrientes consumiendo más forraje (6.25 vs $7.37 \mathrm{~kg} \mathrm{MS} / \mathrm{d}$ ). Estadísticamente se manifestó esta tendencia $(p \geq 0.27)$, debido a que el consumo de FDN impone un freno por la limitada capacidad ruminal que la gestación condiciona. Aun así, las vacas del T2 consumieron más FDN (38 vs $44 \%$ de la dieta; $p<0.05$ ) que las vacas del T1. El incremento en el consumo de forraje hasta donde la FDN permitió, logró compensar el aporte de EM y PM, siendo similares para ambos tratamientos 15 días antes del parto, aunque en el balance de nitrógeno se muestra un déficit de $\mathrm{NH}_{3}$ en el rumen en los dos tratamientos, siendo más acentuado en el T2 (89 vs $86 \%$ del requerido; $\mathrm{p}=0.02$ ).

La primera variable fisiológica que se modifica al inicio de la lactación es la condición corporal. Las vacas del T2 presentaron menor condición corporal que las vacas del T1, consecuencia de mayor utilización de recursos energéticos corporales para soportar la lactación, debido, en parte, a un limitado consumo de MS de las vacas del T2 con respecto al de las vacas del T1 (Tabla 3). Aun cuando hubo un intento por compensar consumiendo más forraje $(p=0.08)$, éste no fue suficiente, perjudicando la relación leche/alimento al producir solo $0.56 \mathrm{~kg}$ de leche por kilogramo de alimento consumido, encareciendo el producto (3.34 vs $4.89 \$ / \mathrm{kg}$ de leche; $p=0.03$ ). 
Tabla 2. Respuesta de vacas secas 15 días antes del parto a dos niveles de suplementación con concentrado.

\begin{tabular}{|c|c|c|c|c|c|c|c|}
\hline \multirow{2}{*}{ Variable } & \multicolumn{3}{|c|}{ Tratamiento 1} & \multicolumn{3}{|c|}{ Tratamiento 2} & \multirow{2}{*}{ Valor $\mathbf{p}$} \\
\hline & Media & DE & EEM & Media & DE & EEM & \\
\hline & \multicolumn{7}{|c|}{ Estado fisiológico } \\
\hline Edad, meses & 113 & 40 & 23 & 90 & 48.9 & 28 & 0.18 \\
\hline Lactancia, número & 5 & 1.7 & 1 & 3.3 & 2.3 & 1.3 & 0.15 \\
\hline Peso cría, kg & 34.4 & 3.61 & 2.1 & 36.1 & 11 & 6.4 & 0.43 \\
\hline Peso corporal, kg & 489 & 46.1 & 27 & 506 & 19.3 & 11 & 0.24 \\
\hline \multirow[t]{2}{*}{ Condición corporal } & 4.5 & 1.26 & 0.73 & 4.0 & 1.63 & 0.94 & 0.32 \\
\hline & \multicolumn{7}{|c|}{ Consumo de materia seca (MS) } \\
\hline Consumo MS observado, kg & 10.7 & 2.27 & 1.3 & 10.5 & 2.51 & 1.4 & 0.45 \\
\hline Consumo concentrado, kg & 4.46 & 0.02 & 0.01 & 3.12 & 0.02 & 0.01 & 0.00 \\
\hline Consumo de forraje, $\mathrm{kg}$ & 6.25 & 2.25 & 1.3 & 7.37 & 2.53 & 1.5 & 0.27 \\
\hline \multirow[t]{2}{*}{ Costo, $\$ / d$} & 51 & 10.3 & 5.9 & 50 & 11.1 & 6.4 & 0.44 \\
\hline & \multicolumn{7}{|c|}{ Consumo de fibra efectiva (eFDN) } \\
\hline Consumo de eNDF, kg & 1.63 & 0.95 & 0.55 & 2.27 & 1.05 & 0.61 & 0.19 \\
\hline Consumo de eNDF, \% dieta & 38 & 5.8 & 3.3 & 44 & 5.6 & 3.2 & 0.05 \\
\hline \multirow[t]{2}{*}{ NDF, \% peso corporal } & 0.81 & 0.26 & 0.15 & 0.93 & 0.34 & 0.20 & 0.27 \\
\hline & \multicolumn{7}{|c|}{ Requerimientos de energía metabolizable (EM) } \\
\hline EM disponible, Mcal/d & 24.4 & 4.53 & 2.6 & 24.5 & 4.69 & 2.7 & 0.52 \\
\hline EM mantenimiento, Mcal/d & 13.6 & 1.16 & 0.67 & 14.2 & 0.42 & 0.24 & 0.22 \\
\hline EM gestación, Mcal/d & 7.2 & 1.6 & 0.93 & 8.13 & 3.74 & 2.20 & 0.38 \\
\hline EM reserva, $\mathrm{Mcal} / \mathrm{d}$ & 3.7 & 4.11 & 2.4 & 2.2 & 3.81 & 2.2 & 0.36 \\
\hline \multirow[t]{2}{*}{ Ganancia de peso, kg/d } & 0.27 & 0.25 & 0.15 & 0.15 & 0.25 & 0.15 & 0.32 \\
\hline & \multicolumn{7}{|c|}{ Requerimientos de proteína metabolizable (PM) } \\
\hline PM disponible, $\mathrm{g}$ & 862 & 110 & 64 & 794 & 129 & 75 & 0.39 \\
\hline PM mantenimiento, $\mathrm{g}$ & 466 & 95.3 & 55 & 491 & 109 & 63 & 0.38 \\
\hline PM gestación, g & 246 & 58.3 & 34 & 283 & 137 & 79 & 0.37 \\
\hline PM reserva, $\mathrm{g}$ & 128 & 146 & 84 & 129 & 196 & 113 & 0.50 \\
\hline PM balance, $\mathrm{g}$ & 22 & 120 & 69 & -48 & 193 & 112 & 0.26 \\
\hline \multirow[t]{2}{*}{ PM requerida, \% } & 106 & 17.2 & 9.9 & 100 & 22.7 & 13 & 0.26 \\
\hline & \multicolumn{7}{|c|}{ Balance de nitrógeno } \\
\hline $\mathrm{NH}_{3}, \%$ requerido & 89 & 12.1 & 7.0 & 86 & 11.2 & 6.4 & 0.02 \\
\hline Urea, costo Mcal/d & 0.17 & 0.07 & 0.04 & 0.09 & 0.08 & 0.05 & 0.21 \\
\hline
\end{tabular}

Tratamiento 1 = consumo de $4.5 \mathrm{~kg}$ de concentrado/d; Tratamiento $2=$ consumo de $3.0 \mathrm{~kg}$ de concentrado/d; DE= desviación estándar; EEM= error estándar de la media.

Con casi $50 \%$ de FDN en la dieta, las vacas del T2 no tuvieron la capacidad de consumir más allá de $3.2 \mathrm{~kg}$ de FDN. El impacto esperado fue no solo menor consumo de energía (33.1 vs 28.2 Mcal; $p=0.03$ ), sino en la distribución de la misma, ya que, aunque ambos tratamientos tuvieron los mismos requerimientos de EM de mantenimiento, las vacas del T2 dispusieron de menor EM para lactación y tendieron a prevenir una caída más drástica de la condición corporal al destinar casi el triple de energía para reserva (Mcal/d) con relación al T1 (1.0 vs 2.77; $p=0.14)$. La consecuencia fue de $4 \mathrm{~kg}$ de leche/d menos en las vacas del T2 (15.7 vs $11.8 ; p=0.03$ ) durante este período.
Ambos tratamientos tuvieron deficiencia de amonio ruminal (números negativos) y como porcentaje del requerido ( 89 y $82 \%$ para T2 y $\mathrm{T} 1$, respectivamente) para los niveles de materia orgánica consumida. No se observaron efectos sobre el contenido de proteína y lactosa de la leche en los tratamientos. Durante el período de 15 a 30 días después del parto (Tabla 4), la condición corporal aparentemente se estabiliza. Se observó cierta tendencia de menor consumo de MS en las vacas del T2 (14.4 vs 12.6 kg/d; $p=0.09)$, dado por el consumo de concentrado, ya que los consumos de forraje y FDN no fueron diferentes entre tratamientos. 
Zárate-Martínez et al - Consumo de forraje y su efecto sobre la reproducción

Tabla 3. Respuesta de vacas lactando los primeros 15 días después del parto a dos niveles de suplementación con concentrado.

\begin{tabular}{|c|c|c|c|c|c|c|c|}
\hline \multirow{2}{*}{ Variable } & \multicolumn{3}{|c|}{ Tratamiento 1} & \multicolumn{3}{|c|}{ Tratamiento 2} & \multirow{2}{*}{ Valor $\mathbf{p}$} \\
\hline & Media & DE & EEM & Media & DE & EEM & \\
\hline & \multicolumn{7}{|c|}{ Estado fisiológico } \\
\hline Peso corporal, kg & 477 & 46.9 & 27.1 & 500 & 25.4 & 14.6 & 0.29 \\
\hline \multirow[t]{2}{*}{ Condición corporal (1 a 9) } & 5.4 & 0.81 & 0.47 & 4.5 & 0.46 & 0.26 & 0.05 \\
\hline & \multicolumn{7}{|c|}{ Consumo de materia seca (MS) } \\
\hline Consumo MS observado, kg & 13.1 & 2.32 & 1.34 & 12.34 & 2.82 & 1.63 & 0.05 \\
\hline Consumo concentrado, kg & 4.5 & 0.04 & 0.02 & 3.1 & 0.01 & 0.01 & 0.00 \\
\hline Consumo de forraje, $\mathrm{kg}$ & 8.6 & 2.35 & 1.36 & 9.24 & 2.83 & 1.64 & 0.08 \\
\hline Costo, $\$ / d$ & 62.3 & 10.7 & 6.2 & 55.8 & 17.5 & 10.1 & 0.13 \\
\hline Relación leche/alimento & 1.01 & 0.35 & 0.20 & 0.56 & 0.41 & 0.24 & 0.00 \\
\hline \multirow[t]{2}{*}{ Costo, $\$ / \mathrm{kg}$ de leche } & 3.34 & 1.62 & 0.93 & 4.89 & 2.09 & 1.21 & 0.03 \\
\hline & \multicolumn{7}{|c|}{ Consumo de fibra efectiva (eFDN) } \\
\hline Consumo de eNDF, kg & 2.5 & 1.06 & 0.61 & 3.2 & 0.85 & 0.49 & 0.01 \\
\hline Consumo de eNDF, \% dieta & 41.7 & 5.17 & 2.98 & 48.6 & 1.1 & 0.63 & 0.05 \\
\hline \multirow[t]{2}{*}{ NDF, \% peso corporal } & 1.17 & 0.44 & 0.25 & 1.21 & 0.27 & 0.16 & 0.37 \\
\hline & \multicolumn{7}{|c|}{ Requerimientos de energía metabolizable (EM) } \\
\hline EM para leche, $\mathrm{kg}$ & 15.7 & 2.06 & 1.19 & 11.8 & 2.07 & 1.20 & 0.03 \\
\hline EM disponible, Mcal/d & 33.1 & 2.45 & 1.41 & 28.2 & 2.83 & 1.64 & 0.03 \\
\hline EM mantenimiento, Mcal/d & 13.2 & 0.85 & 0.49 & 14.0 & 0.63 & 0.37 & 0.17 \\
\hline EM lactación, Mcal/d & 18.9 & 2.44 & 1.41 & 11.4 & 1.98 & 1.14 & 0.01 \\
\hline \multirow[t]{2}{*}{ EM de reserva, Mcal/d } & 1.0 & 1.73 & 1.00 & 2.77 & 3.72 & 2.15 & 0.14 \\
\hline & \multicolumn{7}{|c|}{ Requerimientos de proteína metabolizable (PM) } \\
\hline PM para leche, $\mathrm{kg}$ & 12.8 & 2.44 & 1.41 & 6.1 & 3.23 & 1.86 & 0.00 \\
\hline PM disponible, $\mathrm{g}$ & 1140 & 43.4 & 25.1 & 958 & 78.3 & 45.2 & 0.01 \\
\hline PM para mantenimiento, g & 580 & 92.7 & 53.5 & 590 & 115.9 & 66.9 & 0.28 \\
\hline PM para lactación, g & 655 & 86.4 & 49.9 & 538 & 94.1 & 54.3 & 0.05 \\
\hline PM de reserva, $\mathrm{g}$ & 127 & 109.8 & 63.4 & 260 & 103.8 & 59.9 & 0.00 \\
\hline PM balance, $\mathrm{g}$ & -120 & 121 & 70 & -260 & 104 & 60 & 0.00 \\
\hline \multirow[t]{2}{*}{ PM requerida, \% } & 91 & 9.24 & 5.33 & 79 & 5.86 & 3.38 & 0.01 \\
\hline & \multicolumn{7}{|c|}{ Balance de nitrógeno } \\
\hline $\mathrm{N}$-ureico en leche, $\mathrm{mg} / \mathrm{dL}$ & 0.93 & 1.55 & 0.90 & -0.90 & 1.06 & 0.61 & 0.17 \\
\hline $\mathrm{NH}_{3}$ balance ruminal, $\mathrm{g}$ & -21 & 20.7 & 11.9 & -31 & 5.6 & 3.2 & 0.21 \\
\hline $\mathrm{NH}_{3}$ ruminal, \% requerido & 89 & 11.14 & 6.43 & 82 & 1.53 & 0.88 & 0.21 \\
\hline
\end{tabular}

Tratamiento $1=$ consumo de $4.5 \mathrm{~kg}$ de concentrado/d; Tratamiento $2=$ consumo de $3.0 \mathrm{~kg}$ de concentrado/d; DE= desviación estándar; EEM= error estándar de la media.

Tabla 4. Respuesta de vacas lactando de 15 a 30 días después del parto a dos niveles de suplementación con concentrado.

\begin{tabular}{|c|c|c|c|c|c|c|c|}
\hline \multirow{2}{*}{ Variable } & \multicolumn{3}{|c|}{ Tratamiento 1} & \multicolumn{3}{|c|}{ Tratamiento 2} & \multirow{2}{*}{ Valor $\mathrm{p}$} \\
\hline & Media & DE & EEM & Media & DE & EEM & \\
\hline & \multicolumn{7}{|c|}{ Estado fisiológico } \\
\hline Peso corporal, kg & 477 & 46.9 & 27.1 & 500 & 25.4 & 14.6 & 0.29 \\
\hline \multirow[t]{2}{*}{ Condición corporal (1 a 9) } & 5.13 & 0.92 & 0.53 & 4.64 & 1.83 & 1.06 & 0.25 \\
\hline & \multicolumn{7}{|c|}{ Consumo de materia seca (MS) } \\
\hline Consumo MS observado, kg & 14.4 & 1.52 & 0.879 & 12.6 & 1.04 & 0.598 & 0.09 \\
\hline Consumo concentrado, kg & 4.4 & 0.01 & 0.00 & 3.13 & 0.07 & 0.04 & 0.00 \\
\hline Consumo de forraje, $\mathrm{kg}$ & 10 & 1.53 & 0.88 & 9.46 & 1.09 & 0.63 & 0.31 \\
\hline Costo, $\$ / d$ & 65.2 & 13.1 & 7.56 & 62.5 & 14.3 & 8.27 & 0.31 \\
\hline Relación leche/alimento & 0.82 & 0.116 & 0.067 & 0.48 & 0.234 & 0.135 & 0.11 \\
\hline Costo, $\$ / \mathrm{kg}$ de leche & 4.17 & 0.821 & 0.474 & 5.07 & 1.532 & 0.884 & 0.08 \\
\hline
\end{tabular}


Zárate-Martínez et al - Consumo de forraje y su efecto sobre la reproducción

\begin{tabular}{|c|c|c|c|c|c|c|c|}
\hline & \multicolumn{7}{|c|}{ Consumo de fibra efectiva (eFDN) } \\
\hline Consumo de eNDF, kg & 3.37 & 0.31 & 0.176 & 3.23 & 0.74 & 0.426 & 0.60 \\
\hline Consumo de eNDF, $\%$ dieta & 46.1 & 1.0 & 0.58 & 48.5 & 4.64 & 2.68 & 0.19 \\
\hline \multirow[t]{2}{*}{ NDF, \% peso corporal } & 1.4 & 0.20 & 0.115 & 1.25 & 0.232 & 0.134 & 0.29 \\
\hline & \multicolumn{7}{|c|}{ Requerimientos de energía metabolizable (EM) } \\
\hline EM para leche, $\mathrm{kg}$ & 15.8 & 3.04 & 1.76 & 12.5 & 1.99 & 1.15 & 0.02 \\
\hline EM disponible, Mcal/d & 32.5 & 4.75 & 2.74 & 26.9 & 2.0 & 1.15 & 0.04 \\
\hline EM mantenimiento, Mcal/d & 13.4 & 1.10 & 0.633 & 14.1 & 0.361 & 0.208 & 0.28 \\
\hline EM lactación, Mcal/d & 19 & 3.65 & 2.11 & 12.1 & 1.93 & 1.11 & 0.01 \\
\hline \multirow[t]{2}{*}{ EM de reserva, Mcal/d } & 1.33 & 2.31 & 1.33 & 0.80 & 0.92 & 0.53 & 0.29 \\
\hline & \multicolumn{7}{|c|}{ Requerimientos de proteína metabolizable (PM) } \\
\hline PM para leche, $\mathrm{kg}$ & 11.8 & 1.82 & 1.05 & 6.0 & 2.72 & 1.57 & 0.05 \\
\hline PM disponible, $\mathrm{g}$ & 1170 & 99.2 & 57.2 & 896 & 103.5 & 59.7 & 0.01 \\
\hline PM para mantenimiento, $\mathrm{g}$ & 652 & 48.6 & 28.1 & 604 & 70.9 & 41.0 & 0.21 \\
\hline PM para lactación, g & 658 & 126.8 & 73.2 & 570 & 90 & 52 & 0.03 \\
\hline PM de reserva, $\mathrm{g}$ & 164 & 57.3 & 33.1 & 297 & 137.8 & 79.5 & 0.13 \\
\hline PM balance, g & -164 & 57.3 & 33.1 & -297 & 137.8 & 79.5 & 0.13 \\
\hline \multirow[t]{2}{*}{ PM requerida, \% } & 87.7 & 2.52 & 1.45 & 75.7 & 10.6 & 6.12 & 0.11 \\
\hline & \multicolumn{7}{|c|}{ Balance de nitrógeno } \\
\hline $\mathrm{N}$-ureico en leche, $\mathrm{mg} / \mathrm{dL}$ & 0.73 & 1.19 & 0.69 & -0.27 & 1.66 & 0.96 & 0.04 \\
\hline $\mathrm{NH}_{3}$ balance ruminal, $\mathrm{g}$ & -28.7 & 4.2 & 2.4 & -33.0 & 17.5 & 10.1 & 0.32 \\
\hline $\mathrm{NH}_{3}$ ruminal, \% requerido & 85 & 3.61 & 2.08 & 81 & 9.07 & 5.24 & 0.15 \\
\hline
\end{tabular}

Tratamiento $1=$ consumo de $4.5 \mathrm{~kg}$ de concentrado/d; Tratamiento $2=$ consumo de $3.0 \mathrm{~kg}$ de concentrado/d; DE= desviación estándar; EEM= error estándar de la media.

Después de los 30 días postparto (Tabla 5) se empezó a apreciar recuperación de la condición corporal en las vacas del T2, acompañada de pérdida de peso corporal, pero diferente en las vacas del T1, que mantuvieron el peso corporal, pero con pérdida de condición corporal.

Tabla 5. Respuesta de vacas lactando de 30 a 45 días después del parto a dos niveles de suplementación con concentrado.

\begin{tabular}{|c|c|c|c|c|c|c|c|}
\hline \multirow{2}{*}{ Variable } & \multicolumn{3}{|c|}{ Tratamiento 1} & \multicolumn{3}{|c|}{ Tratamiento 2} & \multirow{2}{*}{ Valor $\mathrm{p}$} \\
\hline & Media & DE & EEM & Media & DE & EEM & \\
\hline & \multicolumn{7}{|c|}{ Estado fisiológico } \\
\hline Peso corporal, kg & 479 & 42.3 & 24.4 & 491 & 41.4 & 23.9 & 0.39 \\
\hline \multirow[t]{2}{*}{ Condición corporal (1 a 9) } & 4.74 & 1.96 & 1.13 & 4.85 & 2.07 & 1.2 & 0.42 \\
\hline & \multicolumn{7}{|c|}{ Consumo de materia seca (MS) } \\
\hline Consumo MS observado, $\mathrm{kg}$ & 15.2 & 2.4 & 1.38 & 12.2 & 1.31 & 0.76 & 0.12 \\
\hline Consumo concentrado, kg & 4.5 & 0.11 & 0.06 & 3.2 & 0.08 & 0.04 & 0.01 \\
\hline Consumo de forraje, $\mathrm{kg}$ & 10.7 & 2.48 & 1.43 & 9.0 & 1.38 & 0.80 & 0.23 \\
\hline Costo, $\$ / d$ & 74.87 & 15.85 & 9.15 & 66.99 & 14.58 & 8.42 & 0.03 \\
\hline Relación leche/alimento & 0.61 & 0.29 & 0.17 & 0.70 & 0.47 & 0.27 & 0.38 \\
\hline \multirow[t]{2}{*}{ Costo, $\$ / \mathrm{kg}$ de leche } & 5.41 & 1.32 & 0.76 & 4.6 & 1.18 & 0.68 & 0.01 \\
\hline & \multicolumn{7}{|c|}{ Consumo de fibra efectiva (eFDN) } \\
\hline Consumo de eNDF, kg & 3.5 & 1.15 & 0.67 & 3.0 & 1.08 & 0.62 & 0.29 \\
\hline Consumo de eNDF, \% dieta & 45.8 & 5.11 & 2.95 & 46.9 & 6.01 & 3.47 & 0.37 \\
\hline \multirow[t]{2}{*}{ NDF, \% peso corporal } & 1.46 & 0.255 & 0.147 & 1.21 & 0.416 & 0.24 & 0.25 \\
\hline & \multicolumn{7}{|c|}{ Requerimientos de energía metabolizable (EM) } \\
\hline EM para leche, $\mathrm{kg}$ & 14.0 & 2.84 & 1.64 & 14.7 & 2.02 & 1.17 & 0.15 \\
\hline EM disponible, Mcal/d & 33.2 & 4.43 & 2.56 & 28.15 & 2.04 & 1.18 & 0.03 \\
\hline EM mantenimiento, Mcal/d & 13.5 & 1.24 & 0.72 & 13.8 & 0.26 & 0.15 & 0.38 \\
\hline EM lactación, Mcal/d & 16.9 & 3.43 & 1.98 & 14.2 & 1.97 & 1.13 & 0.04 \\
\hline EM de reserva, Mcal/d & 2.83 & 1.58 & 0.91 & 0.17 & 0.21 & 0.12 & 0.04 \\
\hline
\end{tabular}




\begin{tabular}{|c|c|c|c|c|c|c|c|}
\hline \multirow[b]{2}{*}{ PM para leche, $\mathrm{kg}$} & \multicolumn{7}{|c|}{ Requerimientos de proteína metabolizable (PM) } \\
\hline & 9.3 & 3.69 & 2.13 & 8.1 & 4.95 & 2.86 & 0.29 \\
\hline PM disponible, $\mathrm{g}$ & 1140 & 167.9 & 96.9 & 951 & 125 & 72.2 & 0.01 \\
\hline PM para mantenimiento, $\mathrm{g}$ & 687 & 124.6 & 71.9 & 580 & 106.2 & 61.3 & 0.19 \\
\hline PM para lactación, g & 585 & 118.7 & 68.5 & 669 & 91.5 & 52.8 & 0.02 \\
\hline PM de reserva, $\mathrm{g}$ & 219 & 150 & 87 & 300 & 179 & 104 & 0.23 \\
\hline PM balance, $\mathrm{g}$ & -200 & 183 & 106 & -300 & 179 & 104 & 0.21 \\
\hline \multirow[t]{2}{*}{ PM requerida, \% } & 86 & 13.1 & 7.54 & 76 & 12.4 & 7.17 & 0.12 \\
\hline & \multicolumn{7}{|c|}{ Balance de nitrógeno } \\
\hline $\mathrm{N}$-ureico en leche, $\mathrm{mg} / \mathrm{dL}$ & 1.2 & 2.05 & 1.18 & -0.63 & 1.92 & 1.11 & 0.01 \\
\hline $\mathrm{NH}_{3}$ balance ruminal, $\mathrm{g}$ & -31.7 & 18.3 & 10.6 & -30.0 & 24.3 & 14.0 & 0.44 \\
\hline $\mathrm{NH}_{3}$ ruminal, $\%$ requerido & 84.7 & 8.02 & 4.63 & 82.7 & 10.97 & 6.33 & 0.31 \\
\hline
\end{tabular}

Tratamiento $1=$ consumo de $4.5 \mathrm{~kg}$ de concentrado/d; Tratamiento $2=$ consumo de $3.0 \mathrm{~kg}$ de concentrado/d; DE= desviación estándar; EEM= error estándar de la media.

El consumo de MS mantuvo la misma tendencia que en el período anterior. Aunque la eficiencia de producción de leche por kilogramo de alimento se recuperó en el T2, de tal forma que el costo de alimentación por $\mathrm{kg}$ de leche producida fue menor ( 5.4 vs $4.6 \$ / \mathrm{kg}$ de leche; $p<0.01$ ), como reflejo de un emparejamiento en la producción de leche (14.0 vs $14.7 \mathrm{~kg} / \mathrm{d} ; \mathrm{p}=0.15)$. En la última etapa del estudio, que corresponde al período de los 45 a los 60 días postparto, previo a la evaluación reproductiva, el estado fisiológico de las vacas mostró algunas modificaciones numéricas (Tabla 6). Las vacas del T1 iniciaron con $489 \mathrm{~kg}$ de peso corporal (PV) al parto y 4.5 de CC, y llegaron a los 60 días posparto con $481 \mathrm{~kg}$ de PV y 5.35 de CC. Es decir, perdieron $8 \mathrm{~kg}$ de PV y ganaron 0.85 unidades de CC. Sin embargo, las vacas del T2 iniciaron con $506 \mathrm{~kg}$ de PV y 4.0 de CC, y a los 60 días postparto pesaron $488 \mathrm{~kg}$ con CC de 4.33 .

Tabla 6. Respuesta de vacas lactando de 45 a 60 días después del parto a dos niveles de suplementación con concentrado.

\begin{tabular}{|c|c|c|c|c|c|c|c|}
\hline \multirow{2}{*}{ Variable } & \multicolumn{3}{|c|}{ Tratamiento 1} & \multicolumn{3}{|c|}{ Tratamiento 2} & \multirow{2}{*}{ Valor $\mathbf{p}$} \\
\hline & Media & DE & EEM & Media & DE & EEM & \\
\hline & \multicolumn{7}{|c|}{ Estado fisiológico } \\
\hline Peso corporal, kg & 481 & 38.3 & 22.1 & 488 & 46.0 & 26.6 & 0.438 \\
\hline \multirow[t]{2}{*}{ Condición corporal (1 a 9) } & 5.35 & 1.86 & 1.07 & 4.33 & 2.12 & 1.22 & 0.096 \\
\hline & \multicolumn{7}{|c|}{ Consumo de materia seca (MS) } \\
\hline Consumo MS observado, kg & 14.5 & 1.41 & 0.81 & 13.1 & 1.94 & 1.12 & 0.080 \\
\hline Consumo concentrado, $\mathrm{kg}$ & 4.5 & 0.08 & 0.049 & 3.1 & 0.08 & 0.048 & 0.001 \\
\hline Consumo de forraje, $\mathrm{kg}$ & 9.97 & 1.49 & 0.86 & 9.98 & 2.00 & 1.15 & 0.493 \\
\hline Costo, $\$ / d$ & 79.2 & 15.61 & 9.01 & 61.1 & 10.59 & 6.11 & 0.073 \\
\hline Relación leche/alimento & 0.71 & 0.514 & 0.297 & 0.60 & 0.52 & 0.30 & 0.206 \\
\hline \multirow[t]{2}{*}{ Costo, $\$ / \mathrm{kg}$ de leche } & 5.77 & 1.098 & 0.634 & 4.19 & 0.506 & 0.292 & 0.070 \\
\hline & \multicolumn{7}{|c|}{ Consumo de fibra efectiva (eFDN) } \\
\hline Consumo de eNDF, kg & 3.13 & 1.185 & 0.684 & 3.37 & 1.29 & 0.745 & 0.211 \\
\hline Consumo de eNDF, \% dieta & 44.4 & 5.89 & 3.4 & 48.0 & 6.29 & 3.63 & 0.026 \\
\hline \multirow[t]{2}{*}{ NDF, \% peso corporal } & 1.34 & 0.291 & 0.168 & 1.36 & 0.53 & 0.307 & 0.449 \\
\hline & \multicolumn{7}{|c|}{ Requerimientos de energía metabolizable (EM) } \\
\hline EM para leche, $\mathrm{kg}$ & 13.9 & 2.89 & 1.67 & 14.6 & 1.47 & 0.85 & 0.326 \\
\hline EM disponible, Mcal/d & 32.9 & 1.42 & 0.820 & 28.4 & 1.03 & 0.597 & 0.003 \\
\hline EM mantenimiento, Mcal/d & 13.5 & 1.17 & 0.674 & 14.1 & 0.611 & 0.353 & 0.291 \\
\hline EM lactación, Mcal/d & 16.7 & 3.5 & 2.02 & 14.1 & 1.47 & 0.85 & 0.128 \\
\hline EM de reserva, $\mathrm{Mcal} / \mathrm{d}$ & 2.77 & 2.42 & 1.4 & 0.17 & 0.21 & 0.12 & 0.095 \\
\hline
\end{tabular}




\begin{tabular}{|c|c|c|c|c|c|c|c|}
\hline \multirow[b]{2}{*}{ PM para leche, $\mathrm{kg}$} & \multicolumn{7}{|c|}{ Requerimientos de proteína metabolizable (PM) } \\
\hline & 9.9 & 6.94 & 4.00 & 7.2 & 5.71 & 3.30 & 0.080 \\
\hline PM disponible, $\mathrm{g}$ & 1136 & 91.9 & 53 & 978 & 112.4 & 64.9 & 0.003 \\
\hline PM para mantenimiento, $\mathrm{g}$ & 651 & 117.8 & 68.0 & 644 & 136.5 & 78.8 & 0.444 \\
\hline PM para lactación, g & 578 & 120.9 & 69.8 & 663 & 66.9 & 38.6 & 0.132 \\
\hline PM de reserva, $\mathrm{g}$ & 184 & 201 & 116 & 335 & 258 & 149 & 0.048 \\
\hline PM balance, $\mathrm{g}$ & -171 & 214 & 124 & -335 & 258 & 149 & 0.051 \\
\hline \multirow[t]{2}{*}{ PM requerida, \% } & 85 & 12.4 & 7.17 & 76 & 15.9 & 9.21 & 0.038 \\
\hline & \multicolumn{7}{|c|}{ Balance de nitrógeno } \\
\hline $\mathrm{N}$-ureico en leche, $\mathrm{mg} / \mathrm{dL}$ & 1.27 & 2.69 & 1.55 & -0.57 & 2.35 & 1.35 & 0.008 \\
\hline $\mathrm{NH}_{3}$ balance ruminal, $\mathrm{g}$ & -28 & 24 & 14 & -212 & 360 & 208 & 0.222 \\
\hline $\mathrm{NH}_{3}$ ruminal, $\%$ requerido & 86 & 9.5 & 5.5 & 84 & 24.3 & 14.1 & 0.435 \\
\hline
\end{tabular}

Tratamiento 1 = consumo de $4.5 \mathrm{~kg}$ de concentrado/d; Tratamiento $2=$ consumo de $3.0 \mathrm{~kg}$ de concentrado/d; DE= desviación estándar; EEM= error estándar de la media.

Lo anterior resultó en una pérdida de $18 \mathrm{~kg}$ de PV y un pobre aumento de 0.33 unidades en la CC. Considerando que las vacas del T2 perdieron $10 \mathrm{~kg}$ de PV más que las vacas del T1, se asume que las vacas del T1 estuvieron en mejor balance energético para reiniciar la actividad reproductiva que las vacas del T2. Los consumos voluntarios de MS y de FDN en este período muestran tendencias, pero no son muy claras para explicar el balance energético, más bien es consecuencia acumulativa de los diferentes niveles de alimentación en los tratamientos a lo largo de todo el estudio. El nivel de concentrado en el T1 favoreció siempre mayor disponibilidad de EM y PM, ya que al comparar estos resultados de alimentación del T1 vs los del T2 para los datos reproductivos, las vacas que recibieron el T1 tuvieron una mayor $(p<0.05)$ tasa de gestación a primer servicio que las vacas que recibieron el T2 (Tabla 7).

Tabla 7. Medias de cuadrados mínimos y sus errores estándar para número de servicios por concepción (NSC), tasa de gestación a primer servicio (TG1), días abiertos (DA) e intervalo entre partos (IEP).

\begin{tabular}{ccccc}
\hline Tratamiento & NSC & TG1 & DA & IEP \\
\hline 1 & 1.4 & 75.2 & 105.4 & 392.6 \\
& $\pm 0.4^{\mathrm{a}}$ & $\pm 16.4^{\mathrm{a}}$ & $\pm 26.5^{\mathrm{a}}$ & $\pm 27.2^{\mathrm{a}}$ \\
& & & & \\
2 & 1.6 & 42.8 & 117.8 & 382.6 \\
& $\pm 0.5^{\mathrm{a}}$ & $\pm 21.1^{\mathrm{b}}$ & $\pm 28.3^{\mathrm{a}}$ & $\pm 31.8^{\mathrm{a}}$ \\
\hline
\end{tabular}

a,bMedias con distinta literal dentro de columna son diferentes $(p<0.05)$.

\section{DISCUSIÓN}

Las vacas durante el inicio de la lactancia aumentan las necesidades de glucosa para la producción de lactosa, pero a falta de ésta, el animal moviliza sustratos glucogénicos (10). Parte de la incompetencia de las vacas del T2 para compensar con forraje el requerimiento de energía que exige la homeorresis de lactación, se debe al contenido de FDN del forraje. El consumo de FDN está en función del tamaño del tubo digestivo del animal. El tamaño del tubo digestivo en bovinos está en función de su peso corporal. Un bovino puede consumir de FDN el $1 \%$ de su peso corporal (11). Vacas en el último mes de gestación tienen limitada esta capacidad de consumo por la ocupación de la cavidad abdominal por el útero. En la Tabla 2 se observa que el consumo de FDN es inferior al $1 \%$ en los dos tratamientos ( 0.81 y $0.93 \%$ del PV). Sin embargo, al inicio de la lactación la demanda energética manifiesta en el T1 exige máximo consumo, aprovechando el factor de distensión ruminal, obteniendo consumos de $1.17,1.4,1.46$ y $1.34 \%$ del PV en los días $15,30,45$ y 60 de lactación (Tablas 3, 4, 5 y 6, respectivamente). Las vacas del T2 al tener menor producción de leche inicial, la exigencia de energía de lactación demandó tendencia diferente de consumo de FDN $(1.21,1.25,1.21$ y $1.36 \%$ del PV) para los mismos períodos de las vacas del T1. Se demuestra que en sistemas doble propósito con alimentación basada en forrajes, proporcionar 4.5 y $3.0 \mathrm{~kg}$ de concentrado energético ( $T 1$ y T2, respectivamente) al inicio de la lactación, provee mayor EM para producción de leche (15.8 vs 12.5 $\mathrm{kg} / \mathrm{d}$; Tabla 4), que tiende a mejorar el consumo de FDN (1.4 vs $1.25 \%$ del PV), maximizando el uso de forraje. 
Al inicio de la lactancia las vacas están en balance energético negativo (BEN), debido a disminuido consumo de MS, lo que provoca una alta movilización de lípidos del tejido adiposo hacia el hígado, provocado por la demanda de producción de leche, compensando así los niveles de glucosa necesarios para equilibrar la energía durante el BEN (12). Las vacas del T1 iniciaron con $489 \mathrm{~kg}$ de PV al parto y 4.5 de CC, y llegaron a los 60 días posparto con $481 \mathrm{~kg}$ de PV y 5.35 de CC. Es decir, perdieron $8 \mathrm{~kg}$ de PV y ganaron 0.85 unidades de CC. Sin embargo, las vacas del T2 iniciaron con $506 \mathrm{~kg}$ de PV y 4.0 de CC, y a los 60 días postparto pesaron 488 $\mathrm{kg}$ con CC de 4.33. Lo anterior resultó en una pérdida de $18 \mathrm{~kg}$ de $\mathrm{PV}$ y un pobre aumento de 0.33 unidades en la CC. Con esta información y utilizando ecuaciones reportadas previamente (13) se calculó la energía retenida a los 60 días de lactación por cada tratamiento, resultando que las vacas del T1 retuvieron $132.6 \mathrm{Mcal}$ de ENL de la dieta y las vacas del T2 solo 9.2. Las vacas del T1 tuvieron mejor balance energético para reiniciar la actividad reproductiva que las vacas del $\mathrm{T} 2$.

Diversos estudios demuestran que la síntesis de glucosa en las vacas se puede estimular con el uso de precursores tales como propionato (14) o propilenglicol (15), por lo que ésta puede ser una opción para corregir el BEN y reducir el consumo de FDN. Los resultados de este trabajo muestran que la disponibilidad de proteína metabolizable (PM) fue más crítica todavía para ambos grupos. En el T1, de los $15.7 \mathrm{~kg}$ de leche producida, 12.8 $\mathrm{kg}$ fueron cubiertos por la proteína de la dieta; en el T2, de los $11.8 \mathrm{~kg}$ de leche producida, solo $6.1 \mathrm{~kg}$ fueron proporcionados por la proteína de la dieta.

La PM de mantenimiento es indistinta entre tratamientos por razones de sobrevivencia, pero se observa un trueque entre la PM de lactación y la de reserva entre tratamientos, siendo 117 g mayor la PM que se destina para lactación en el T1, y $133 \mathrm{~g}$ mayor la PM para reserva en el T2. Esto quizás porque el estrés proteico estuvo más acentuado en el T2, ya que solo se cubrió el $79 \%$ de sus requerimientos de PM, y en el T1 el $91 \%$. La proteína de la dieta representa del 42 al $50 \%$ del costo total de las raciones de productos lácteos (16) y juega un papel importante en la rentabilidad del sistema de producción, ya que afecta el rendimiento de las vacas lecheras y el medio ambiente (17).
Una estrategia metabólica directa es tender a secretar menos $\mathrm{N}$-ureico en leche $(\mathrm{mg} / \mathrm{dL})$, el cual fue de 0.93 y $-0.90(p=0.17)$ para $T 1$ y T2, respectivamente. En una investigación con vacas de producción media en Nueva Zelanda, el aumento de los suministros de PM no modificó la ingesta de MS, sin embargo, aumentó linealmente la producción de proteína, grasa y lactosa de la leche. De manera similar, la producción de leche corregida por grasa aumentó linealmente $(9.3 \%)$, debido a un aumento tanto en el rendimiento (5.2\%) como en el contenido de grasa de la leche (7.8\%). La eficiencia del nitrógeno de la leche (ENL) disminuyó de 0.26 a 0.20 , mientras que la eficiencia metabólica de PM disminuyó de 0.70 a 0.60 en suministros de PM bajo a alto, respectivamente (18).

Diferentes modelos de requerimientos de nutrientes usan diferentes eficiencias de PM para predecir los requerimientos de proteína en vacas lecheras lactantes; por ejemplo, el modelo NRC (19) propone una eficiencia de PM de 0.67, que es mayor que la del INRA (20) y CNCPS (13), que proponen 0.64 y 0.65 , respectivamente. Cabe resaltar el esfuerzo que realizaron las vacas de este estudio para ingerir FDN (1.40 vs $1.25 \%$ del peso corporal) por arriba de su límite físico ( $1 \%$ del peso corporal), al recurrir al beneficio de la distención ruminal.

Dada la condición del tratamiento, las vacas del T1 lograron $(p<0.05)$ consumir más EM/d (32.5 vs 26.9 Mcal), derivar más EM para lactación (19 vs $12.1 \mathrm{Mcal}$ ) y producir más leche (15.8 vs $12.5 \mathrm{~kg} / \mathrm{d}$ ). Sin embargo, el primer nutrimento limitante sigue siendo la PM, donde las vacas del T1 y T2 $(p<0.05)$ dispusieron de 1170 y $896 \mathrm{~g} / \mathrm{d}$, de los cuales destinaron para lactación 658 y $570 \mathrm{~g} / \mathrm{d}$, suficientes para producir apenas 11.8 y $6.0 \mathrm{~kg}$ de leche/d. Ambos tratamientos estuvieron en balance negativo de PM, al cubrir solamente el 88 y $76 \%$ del requerimiento. La consecuencia para el T2 es que tuvo que reducir el $\mathrm{N}$-ureico en leche ( 0.73 vs $-0.27 ; \mathrm{p}=0.04)$ para economizar $\mathrm{N}$.

Se ha demostrado que aumentar los suministros de proteínas en la dieta por debajo del requisito, aumenta el rendimiento de proteínas de la leche, sin embargo, la ENL disminuyó $(14,21)$. Parte de la deficiencia de PM se debió a una reducción en la síntesis de proteína microbiana debido a que solo se proporcionó alrededor del $80 \%$ del $\mathrm{NH}_{3}$ requerido para la materia orgánica fermentable disponible en rumen. 
Aun cuando las vacas del T1 consumieron más EM (33.2 vs $28.1 \mathrm{Mcal} / \mathrm{d}$ ), la diferencia no la derivaron toda hacia lactación, sino en partes iguales hacia reserva. Debe existir alguna señalización (quizás hormonal) que le indique a la vaca como repartir los nutrientes para lactación y reserva, dependiendo del estado fisiológico y nutricional en que se encuentre. Algunos autores (22) explican que dietas más glucogénicas (como es el caso del T1), disminuyen el gasto energético en leche, y tienden a estimular la partición de la energía hacia reservas corporales, y mejorar el balance energético y el comportamiento reproductivo en comparación con dietas lipogénicas (menor densidad energética en la dieta) por tener menor concentración plasmática de BHBA, NEFA y TAG en hígado. Esta señalización también podría explicar cómo es que las vacas del T2 derivaron más PM para lactación que las vacas del T1 (585 vs $669 \mathrm{~g} ; \mathrm{p}=0.02$ ) durante este período, manteniendo negativo todavía el $\mathrm{N}$-ureico en leche ( 1.2 vs $-0.63 \mathrm{mg} / \mathrm{dL})$ y permitiendo mantener baja además la excreción de $\mathrm{NH}_{3}$ (30 vs $12 \mathrm{~g} / \mathrm{d}$ ).

Las vacas del T1 perdieron menos CC; esta ventaja se vio reflejada en una mayor $(p<0.05)$ tasa de gestación a primer servicio. Este comportamiento indica que las vacas del T1, aunque no derivaron suficiente energía proveniente de gluconeogénesis para mayor lactogénesis, si aportaron la glucosa necesaria que ocupan los folículos ováricos para su desarrollo y madurez, que junto con un protocolo de IATF, culminó en que más de la mitad de las vacas quedaran gestantes a primer servicio a diferencia del T2, con el cual menos de la mitad respondieron al tratamiento de sincronización de la ovulación a los 70 días posparto.

Los problemas reproductivos en el trópico húmedo están condicionados por un sinnúmero de factores: las condiciones ambientales, el manejo, la falta de energía en la dieta, la salud, la infraestructura y los genotipos utilizados; todos estos influyen para no lograr una eficiente reproducción (23). Es difícil determinar una base causal para la disminución de la fertilidad, ya que la genética y el medio ambiente han cambiado notablemente a lo largo de las últimas décadas. Aunque parte de la disminución de la fertilidad observada está asociada con la selección genética para una mayor producción de leche y la heredabilidad de la reproducción es mucho más baja (24).
Influencias nutricionales durante el período de transición ( \pm 4 semanas de parto) pueden ser de particular importancia (25), pero está claro que el efecto de la dieta sobre la fertilidad durante este período es complejo y multifactorial, por lo que el uso de IATF como alternativa, permitió servir a las vacas poco después del periodo de espera voluntario, independientemente del estado de ciclicidad en que se encontraban, aumentando la tasa de servicio (26). En este sentido, las técnicas hormonales usadas para acelerar la presentación de la ciclicidad ovárica durante el posparto tienen un gran impacto en la producción bovina $(26,27)$.

Se recomienda que en el período de 45 a 60 días se incremente la concentración de EM en la ración. Paralelamente, se debe reducir la concentración de FDN en la ración, porque quedó demostrado que las vacas tienen un límite ( $1 \%$ de su PV) para consumir fibra. Arriba de este porcentaje se compromete el consumo de MS y, por tanto, de nutrientes. Debido a que el incremento de EM en la ración de vacas cruzadas en el trópico se ve limitado por un alto consumo de FDN, la administración de precursores glucogénicos durante el periodo de transición podría resultar en un efecto positivo.

Otra observación de este estudio es que la PM siempre fue deficiente durante la lactación. En condiciones tropicales, la PM siempre será el primer nutrimento limitante, dado que los pastos tropicales son bajos en proteína (28). De acuerdo con los resultados bajo las condiciones del estudio, para producir $14 \mathrm{~kg}$ de leche/d se requieren mínimo 30 Mcal de EM y $1,350 \mathrm{~g}$ de PM. Otra de las observaciones en el T2 es que la deficiencia de PM repercutió en menor contenido de $\mathrm{N}$ en leche, deficiencia que impacta directamente a la industria quesera, que es el principal destino de la leche en la región. Por otro lado, apoyando con amamantamiento restringido ( $8 \mathrm{~h}$ posteriores al ordeño) y destete temporal de $72 \mathrm{~h}$ a partir del retiro del CIDR al aplicar el protocolo hormonal de IATF, se maximizó también el comportamiento reproductivo de vacas en el trópico al implementar un programa de sincronización de la ovulación a los 70 días posparto. Se obtuvo hasta un $75 \%$ de tasa de preñez a primer servicio con períodos abiertos de 105 a 117 d y períodos interparto de 13 a 14 meses, parámetros que impactarían también en la eficiencia reproductiva del trópico de México. 
Es evidente que la importancia de buscar un balance de energía y proteínas en la lactancia temprana mejorará el balance energético y la fertilidad, y sugiere que el almidón y los azúcares (dietas glucogénicas) pueden tener diferentes efectos sobre la proporción de vacas que se preñan con inseminación artificial (22).

En conclusión, al inicio de la lactación la demanda energética de las vacas en el T1 exige máximo consumo, aprovechando el factor de distensión ruminal. Se recomienda que en el período de 45 a 60 días posparto se incremente la concentración de EM. Se debe reducir la concentración de FDN en la ración, porque quedó demostrado que las vacas tienen un límite de $1 \%$ de su PV para consumir fibra. El incremento de EM en la ración de vacas cruzadas en el trópico se ve limitado por un alto consumo de FDN, por lo que la administración de precursores glucogénicos durante el periodo de transición podría resultar en un efecto positivo. Es necesario reducir el consumo de fibra. Debido a que la PM de balance siempre fue deficiente, es necesario enfocar estudios con aporte de aminoácidos específicos a partir de los primeros 15 días de la lactación. La disminución de la FDN en la dieta de 44 a $38 \%$ y la aplicación del protocolo hormonal de IATF disminuye el período abierto a $105 \mathrm{~d}$ en vacas lactando en el trópico.

\section{Conflicto de interés}

Los autores no tienen ningún conflicto de intereses.

\section{Agradecimientos}

El presente trabajo se realizó con financiamiento de recursos fiscales INIFAP asignados al proyecto "Alimentación funcional y protocolos hormonales para aumentar la fertilidad y cosecha de becerros en el trópico de México".

\section{REFERENCIAS}

1. Wankhade $P R$, Manimaran A, Kumaresan A, Jeyakumar S, Ramesha KP, Sejian V, Rajendran D, Varghese MR. Metabolic and immunological changes in transition dairy cows. Vet World. 2017; 10(11):13671377. https://doi.org/10.14202/ vetworld.2017.1367-1377

2. Shimada MA. Nutrición animal. $1^{\text {a }}$ ed. México, D.F.: Trillas; 2003. https://es.scribd. com/doc/130788109/Nutricion-AnimalShimada

3. Allen MS. Control of feed intake by hepatic oxidation in ruminant animals: integration of homeostasis and homeorhesis. Animal. 2020; 14(S1):s55-s64. https://doi. org/10.1017/S1751731119003215

4. García E. Modificaciones al sistema de clasificación climática de Köppen. $5^{a}$ ed. México, D.F.: Instituto de Geografía-UNAM; 2004. https://publicaciones.igg.unam.mx/ index.php/ig/catalog/view/83/82/251-1
5. Van Amburgh ME, Collao-Saenz EA, Higgs RJ, Ross DA, Recktenwald EB, Raffrenato E, Chase LE, Overton TR, Mills JK, Foskolos A. The Cornell Net Carbohydrate and Protein System: Updates to the model and evaluation of version 6.5. J Dairy Sci. 2015; 98(9):6361-6380. https://doi.org/10.3168/ jds.2015-9378

6. Van Soest PJ, Robertson JB, Lewis BA. Methods for dietary fiber, neutral detergent fiber, and nonstarch polysaccharides in relation to animal nutrition. J Dairy Sci. 1991; 74(10):3583-3597. https://doi. org/10.3168/jds.S0022-0302(91)78551-2

7. Licitra G, Hernandez TM, Van Soest PJ. Standardization of procedures for nitrogen fractionation of ruminant feeds. Anim Feed Sci Technol. 1996; 57(4):347-358. https:// doi.org/10.1016/0377-8401(95)00837-3 
8. Higgs RJ, Chase LE, Ross DA, Van Amburgh ME. Updating the Cornell Net Carbohydrate and Protein System feed library and analyzing model sensitivity to feed inputs. J Dairy Sci. 2015; 98(9):6340-6360. https:// doi.org/10.3168/jds.2015-9379

9. SAS. SAS/STAT. Version 9.3. 4th ed. SAS Institute: Cary, USA; 2011. https://support. sas.com/en/software/sas-stat-support.html

10. Aschenbach JR, Kristensen NB, Donkin SS, Hammon HM, Penner GB. Gluconeogenesis in dairy cows: the secret of making sweet milk from sour dough. IUBMB Life. 2010; 62(12):869-877. https://doi.org/10.1002/ iub.400

11. Van Soest PJ. Nutritional ecology of the ruminant. Ithaca, New York, USA: Cornell University Press; 1994. https://www.jstor. org/stable/10.7591/j.ctv5rf668

12. Bjerre-Harpoth $V$, Storm AC, Vestergaard M, Larsen M, Larsen T. Effect of postpartum propylene glycol allocation to overconditioned Holstein cows on concentrations of milk metabolites. J Dairy Res. 2016; 83(2):156-164. https://doi.org/10.1017/ $\underline{\text { S0022029916000145 }}$

13. Fox DG, Van Amburgh ME, Tylutki TP. Predicting requirements for growth, maturity, and body reserves in dairy cattle. J Dairy Sci. 1999; 82(9):1968-1977. https://doi. org/10.3168/jds.S0022-0302(99)75433-0

14. Lammoglia-Villagómez $M$, Cabrera-Nuñez A, Alarcón-Zapata M, Rojas-Ronquillo R, Chagoya-Fuentes J, Daniel-Rentería I. Beneficios del propilenglicol en el periparto en cetosis subclínica y parámetros productivos en el trópico veracruzano. Abanico Vet. 2019; 9:1-7. http://dx.doi.org/10.21929/ abavet2019.97

15. Lien TF, Chang LB, Horng YM, Wu CP. Effects of propylene glycol on milk production, serum metabolites and reproductive performance during the transition period of dairy cows. Asian-Aust J Anim Sci. 2010; 23(3):372-378. https://doi.org/10.5713/ajas.2010.60620
16. St-Pierre N. The cost of nutrients, comparison of feedstuffs prices and the current dairy situation. Buckeye Dairy News. 2012; 14(2). https://dairy.osu.edu/newsletter/buckeyedairy-news/volume-14-issue-2/costsnutrients-comparison-feedstuffs-prices-and

17. Lee J, Seo J, Lee SY, Ki KS, Seo S. Metaanalysis of factors affecting milk component yields in dairy cattle. J Anim Sci Technol. 2014; 56:5 https://doi.org/10.1186/2055$\underline{0391-56-5}$

18. Imran $M$, Pasha $T N$, Shahid $M Q$, Babar I, Naveed UI Haque M. Effect of increasing dietary metabolizable protein on nitrogen efficiency in Holstein dairy cows. AsianAustralas J Anim Sci. 2017; 30(5):660-665. https://doi.org/10.5713/ajas.16.0564

19. National Research Council. Nutrient requirements of dairy cattle. 7th ed. Washington, DC: National Academy Press; 2001. https://profsite.um.ac.ir/ kalidari/ software/NRC/HELP/NRC\%202001.pdf

20. Salah N. Nutrition of goats, sheep and cattle in tropical and warm conditions "Evaluation of energy and protein requirements and animal responses to diet. Evaluation of INRA system to predict nutritive value of forage resources". Agricultural sciences. AgroParisTech; 2015. https://tel.archivesouvertes.fr/tel-02004274/document

21. Brun-Lafleur L, Delaby L, Husson F, Faverdin P. Predicting energy $x$ protein interaction on milk yield and milk composition in dairy cows. J Dairy Sci. 2010; 93(9):4128-4143. https://doi.org/10.3168/jds.2009-2669

22. Van Knegsel ATM, van den Brand $H$, Dijkstra J, Kemp B. Effects of dietary energy source on energy balance, metabolites and reproduction variables in dairy cows in early lactation. Theriogenology. 2007; 68:S274-S280. https://doi.org/10.1016/j. theriogenology.2007.04.043 
23. Moyano JC, López JC, Vargas J, Quinteros OR, Marini PR. Plasmaspiegel von LH (luteinisierendes hormon), brunstsymptome und qualität der gelbkörper in verschiedenen protokollen, zur synchronisation der brunst in Brown-Swiss-Milchrindern. Züchtungskunde. 2015; 87(4):265-271. https://www. zuechtungskunde.de/Plasmaspiegelvon-LH- Iuteinisierendes-HormonBrunstsymptome-und-Qualitaet-derGelbkoerper-in-verschiedenenProtokollenzur-Synchronisation-derBrunstinBrownSwiss-Milchrinder,QUIEPTQ3NzIzNzUmTUI EPTY5MTU4.html

24. Berry DP, Wall E, Pryce JE. Genetics and genomics of reproductive performance in dairy and beef cattle. Animal. 2014; 8(S1):105-121. https://doi.org/10.1017/ $\underline{\mathrm{S} 1751731114000743}$

25. Lean IJ, Van Saun R, DeGaris PJ. Energy and protein nutrition management of transition dairy cows. Vet Clin North Am Food Anim Pract. 2013; 29(2):337-366 https://doi. org/10.1016/j.cvfa.2013.03.005
26. Bó GA, Baruselli PS. Synchronization of ovulation and fixed-time artificial insemination in beef cattle. Animal. 2014; 8(Suppl 1):144-150. https://doi. org/10.1017/S1751731114000822

27. Baruselli PS, Ferreira RM, Sá Filho MF, Bó GA. Using artificial insemination $v$. natural service in beef herds. Animal. 2018; 12(Suppl 1):S45-S52. https://doi. org/10.1017/S175173111800054X

28. Juarez-Lagunes FI, Fox DG, Blake RW, Pell AN. Evaluation of tropical grasses for milk production by dual-purpose cows in tropical Mexico. J Dairy Sci. 1999; 82:21362145. https://doi.org/10.3168/jds.S0022$\underline{0302(99) 75457-3}$ 